\title{
Measuring radial flow of partonic and hadronic phases in relativistic heavy ion collision.
}

\author{
Jajati K Nayak and Jan-e Alam \\ Variable Energy Cyclotron Centre, 1/AF, Bidhan Nagar, Kolkata - 700064
}

(Dated: November 12, 2018)

\begin{abstract}
It has been shown that the thermal photon and the lepton pair spectra can be used to estimate the radial velocity of different phases of the matter formed in nuclear collisions at ultra-relativistic energies. We observe a non-monotonic variation of the flow velocity with invariant mass of the lepton pair which is indicative of two different thermal dilepton sources at early and late stage of the dynamically evolving system. We also show that the study of radial velocity through electromagnetic probes may shed light on the nature of the phase transition from hadrons to QGP.

PACS numbers: 25.75.-q,25.75.Dw,24.85.+p
\end{abstract}

\section{INTRODUCTION}

The numerical simulation of QCD equation of state (EoS) predicts that nuclear matter at high density and/or temperature are composed of quarks and gluons due to asymptotic freedom and screening of colour charges [1, 2, 3]. Enormous experimental efforts have been made to produce such a partonic state of a matter, called Quark Gluon Plasma (QGP) by colliding nuclei at ultra-relativistic energies. Careful theoretical investigations have been performed to understand the existing experimental data 44 and predictions for the forthcoming experiments [5] have also been made.

The hot and dense matter formed in the partonic phase after ultra-relativistic heavy ion collisions expands in space and time due to high internal pressure. Consequently the system cools and reverts back to hadronic matter from the partonic phase. Initially (when the thermal system is just born) the entire energy of the system is thermal in nature and with progress of time some part of the thermal energy gets converted to the collective (flow) energy. In other words during the expansion stage the total energy of the system is shared by the thermal as well as collective degrees of freedom. The evolution of the collectivity within the system is sensitive to the EoS. Therefore, the study of the collectivity in the system formed after nuclear collisions will be useful to shed light on the $\operatorname{EoS}[6,7,8]$ of the strongly interacting system at high temperatures and densities.

It is well known that the average magnitude of radial flow can be extracted from the transverse momentum $\left(p_{T}\right)$ spectra of the hadrons. However, hadrons being strongly interacting objects can bring the information of the state of the system when it is too dilute to support collectivity. On the other hand electromagnetic (EM) probes i.e. photons and dileptons are produced and emitted from each space time points. Therefore, estimating radial flow from the EM probes will shed light on the time evolution of the collectivity in the system. This is demonstrated by NA60 collaboration [9] through dilepton measurements in In+In collisions at SPS energy. The slope of the transverse mass spectrum of lepton pairs, $T_{\mathrm{e} f f}$ of invariant mass $M$ can be related to the space-timed averaged quantities like radial flow velocity $v_{\mathrm{r}}$ and temperature $T_{\mathrm{a} v}$ as $T_{\mathrm{eff}} \sim T_{\mathrm{av}}+M v_{\mathrm{r}}^{2}$. $T_{\text {eff }}$ estimated from dilepton spectra [9] shows a different kind of behaviour [10, 11, 12, 13, 14, 15] as compared to that from hadronic spectra. The effective temperature extracted from transverse mass spectra of dileptons increases linearly with invariant mass $M$ up to $\rho$-peak and then falls (the PHENIX data does not show this trend [16]). In a recent work [17] we have shown that the ratio $\left(R_{\mathrm{em}}\right)$ of the $p_{T}$ spectra of photons to lepton pairs has an advantage over the individual spectra because some of the uncertainties or model dependence pertaining to the individual spectra gets canceled in the ratio. Hence the ratio can be used as an efficient tool to understand the state of an expanding system. In the present work we focus on the extraction of the radial flow from $R_{\mathrm{e} m}$. We also argue that the simultaneous measurements of photons and dileptons will enable us to estimate the value of $v_{\mathrm{r}}$ for various invariant mass windows of the lepton pairs. The $v_{\mathrm{r}}$ obtained from the analysis of both the spectra vary with $M$ non-monotonically. Such a behaviour may be interpreted as due to the presence of two different kinds of thermal sources of lepton pairs of the expanding system.

The paper is organized as follows. In section II the ratio of thermal photon and dilepton productions has been discussed. In section III the evolution dynamics of the hot fireball system with specific initial conditions and EoS is outlined. The discussions in sections II and III will be very brief as the details are available elsewhere [17]. The results are presented in 
section IV. Finally section $\mathrm{V}$ is devoted to summary and discussions.

\section{ELECTROMAGNETIC PROBES}

The ratio, $R_{\mathrm{e} m}$ of the $p_{T}$ spectra of thermal photons to dileptons can be written as follows [17]:

$$
R_{e m}=\frac{\frac{d^{2} N_{\gamma}}{d^{2} p_{T} d y}}{\frac{d^{2} N_{\gamma}^{\star}}{d^{2} p_{T} d y}}=\frac{\sum_{i} \int_{i}\left(\frac{d^{2} R_{\gamma}}{d^{2} p_{T} d y}\right)_{i} d^{4} x}{\sum_{i} \int_{i}\left(\frac{d^{2} R_{\gamma^{*}}}{d^{2} p_{T} d y d M^{2}}\right)_{i} d M^{2} d^{4} x .}
$$

The numerator (denominator) is the invariant momentum distribution of the thermal photons (lepton pairs). In Eq. $1 p_{T}, y$ and $M$ denote the transverse momentum, rapidity and the invariant mass of the lepton pair. The summation in Eq. 1 runs over all phases through which the system passes during the expansion. $\left(d^{2} R / d^{2} p_{T} d y\right)_{i}$ and $\left(d^{2} R / d^{2} p_{T} d y d M^{2}\right)_{i}$ are the static rates of photon and dilepton productions from the phase $i$, which is convoluted over the expansion dynamics through the spacetime integration over $d^{4} x$. The integration over $M$ is done by selecting invariant mass windows $M_{\min } \leq M \leq M_{\max }$ appropriately and we define $<M>=\left(M_{\min }+M_{\max }\right) / 2$.

The rate of thermal dilepton production per unit space-time volume per unit four momentum volume is given by [18, 19, 20, 21]

$$
\frac{d R}{d^{4} p}=\frac{\alpha}{12 \pi^{4} p^{2}} L\left(p^{2}\right) \operatorname{I} m \Pi_{\mu}^{R \mu} f_{B E}
$$

where $\alpha$ is the EM coupling constant, $\operatorname{I} m \Pi_{\mu}^{\mu}$ is the imaginary part of the retarded photon self energy and $f_{B E}(E, T)$ is the thermal phase space factor for Bosons. $L\left(p^{2}\right)=\left(1+\frac{2 m^{2}}{p^{2}}\right) \sqrt{1-4 \frac{m^{2}}{p^{2}}}$ arises from the final state leptonic current involving Dirac spinors of mass $m$. The real photon production rate can be obtained from the dilepton emission rate by replacing the product of EM vertex $\gamma^{\star} \rightarrow l^{+} l^{-}$, the term involving final state leptonic current and the square of the (virtual) photon propagator by the polarization sum for the real photon. For an expanding system $E$ should be replaced by $u_{\mu} p^{\mu}$ where $p^{\mu}$ and $u^{\mu}$ are the four momentum and the four velocity respectively.

\section{A. Thermal photons}

The photon production rate has been evaluated by various authors [22] using Hard Thermal Loops [23] approximations. The complete calculation of emission rate of photons from QGP to order $\mathrm{O}\left(\alpha, \alpha_{s}\right)$ has been done by resuming ladder diagrams in the effective theory [24]. This rate of production has been considered in the present work. A set of hadronic reactions with all isospin combinations have been considered for the production of photons 25, 26, 27] from hadronic matter. The effect of hadronic dipole form factors has been taken into account in the present work. We have checked that the high $p_{T}$ $(\sim 2-3 \mathrm{GeV})$ part of the thermal photon spectra is dominated by the contributions from QGP phase for large initial temperature.

\section{B. Thermal dileptons}

The lowest order process producing lepton pairs is $q$ and $\bar{q}$ annihilation. For a finite temperature QCD plasma, the correction of order $\alpha_{s} \alpha^{2}$ to the lowest order rate of dilepton production has been calculated in [28, 29], which is considered in the present work. For the low $M$ dilepton production from the hadronic phase we consider the decay of light vector mesons $\rho, \omega$ and $\phi$ as considered in [17]. The continuum part of the vector mesons spectral functions have been included in the present work 30 , 31].

It is well known that the contributions from the QGP phase dominates the $M$ spectra of the lepton pairs below $\rho$-peak and above the $\phi$-peak if no thermal effects of the spectral functions of the vector mesons (see [30, 32, 33] for review) are considered.

\section{EVOLUTION DYNAMICS}

In the collision of two energetic heavy ions a large amount of energy is dumped into a small volume. The space-time evolution of the matter has been studied using ideal relativistic hydrodynamics [34] with longitudinal boost invariance [35] and cylindrical symmetry. The initial energy density $\left(\epsilon\left(\tau_{i}, r\right)\right)$ and radial velocity $\left(v\left(\tau_{i}, r\right)\right)$ profiles are same as our earlier studies [17]. The value of transition temperature $\left(T_{c}\right)$ is taken as $192 \mathrm{MeV}$ as obtained in lattice QCD calculations [36]. Although a much lower value of $T_{c}$ is also predicted in [37]. However, we have found that the dependence of $R_{\mathrm{e} m}$ on $T_{c}$ is weak. In a first order phase transition scenario we use Bag EoS for the QGP phase and for the hadronic phase all the resonances with mass $\leq 2.5 \mathrm{GeV}$ have been considered [38].

In the present work we have considered the initial and freeze out conditions that reproduced the hadrons [39], the photon [40] and dilepton spectra for RHIC energy [41]. The values of initial thermalization time, $\tau_{i}=0.2 \mathrm{fm} / \mathrm{c}$, initial temperature $T_{i}=400 \mathrm{MeV}$ and the freeze-out temperature $T_{F}=120 \mathrm{MeV}$ have been taken as the input to the calculation. For LHC we have taken $T_{i}=700 \mathrm{MeV}$, 


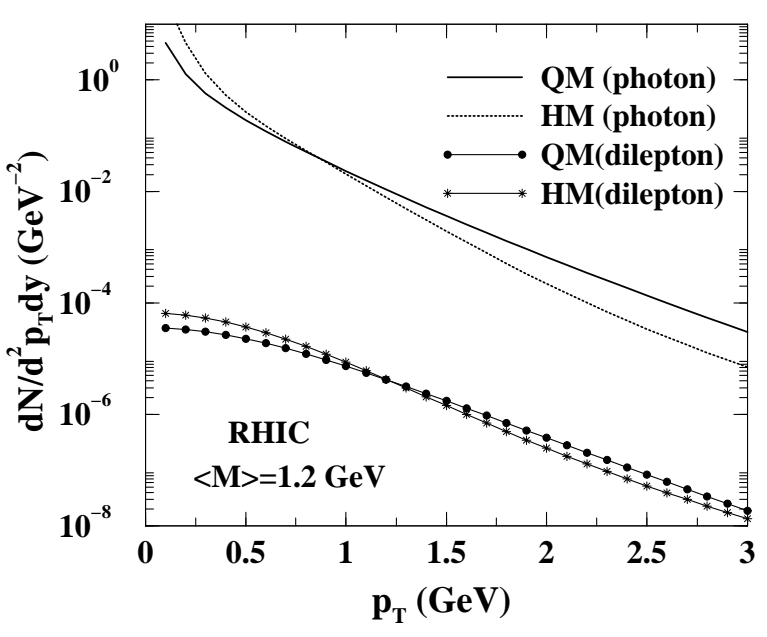

FIG. 1: The $p_{T}$ spectra of photons and dileptons from hadronic and quark matter at RHIC energy. The dilepton spectra is obtained by doing $M$ integration from $M=1.0 \mathrm{GeV}$ to $1.4 \mathrm{GeV}$

$\tau_{i}=0.08 \mathrm{fm} / \mathrm{c}$ which gives the hadron multiplicity $d N / d y=2100[5]$.

\section{RESULTS AND DISCUSSION}

In Fig. 1 the photon and dilepton spectra have been displayed for RHIC conditions. Results indicate that the photon spectra from QGP dominates over its hadronic counterpart for $p_{T}>1.5 \mathrm{GeV}$. The dilepton from QGP and hadrons are comparable in magnitude for entire range of $p_{T}$ for $M \sim 1.2 \mathrm{GeV}$ (this is because of the inclusion of the continuum of the vector meson spectral functions [30, 31] without the continuum the quark matter part dominates). However, for $M \sim 0.75 \mathrm{GeV}$ the dileptons from the hadronic matter are overwhelmingly large compared to quark matter contributions (not shown in the figure). Therefore, an appropriate selection of $p_{T}$ and $M$ will be very useful to characterize a particular phase of the system.

Now we consider the variation of the ratio, $R_{e m}$ as a function of $p_{T}$ for different invariant mass windows. The results are shown in the Fig. 2 and Fig. 3 below. The variation of $R_{\mathrm{e} m}$ with respect to $p_{T}$ can be parametrized as follows:

$$
R_{e m} \equiv A_{3}\left[\frac{m_{T}}{p_{T}}\right]^{B_{3}} \exp \left[C_{3}\left(m_{T}-p_{T}\right)\right]
$$

where $A_{3}, B_{3}$ and $C_{3}$ are constants and $M_{T}$, the transverse mass of the lepton pair is defined as, $M_{T}=\sqrt{p_{T}^{2}+M^{2}}$. It is observed that the ratio decreases sharply and reaches a plateau beyond
$p_{T}>1.5 \mathrm{GeV}$. This behaviour of $R_{\mathrm{e} m}$ as a function of $p_{T}$ can be understood as follows: (i) for $p_{T} \gg M M_{T} \sim p_{T}$ and consequently $R_{\mathrm{e} m} \sim A_{3}$ giving rise to a plateau at large $p_{T}$. The height of the plateau is sensitive to the initial temperature of the system [17]. (ii) For $p_{T}<M R_{\mathrm{e} m} \sim$ $\exp \left(-p_{T} / T_{\mathrm{e} f f}\right) / p_{T}^{\mathrm{B}_{3}}$ indicating a decrease of the ratio with $p_{T}\left(\right.$ at low $p_{T}$ ) as observed in the Fig. 2 and Fig. 3,

To indicate the effect of the radial flow velocity, $v_{\mathrm{r}}$ we have evaluated the $R_{\mathrm{e} m}$ with and without radial flow (see Fig. 2 and Fig. 31). In case of vanishing radial flow the ratio can be parametrized as follows:

$$
R^{1}{ }_{\text {em }} \equiv A_{1}\left[\frac{m_{T}}{p_{T}}\right]^{B_{1}} \exp \left[C_{1}\left(m_{T}-p_{T}\right)\right]
$$

Here $C_{1}$ contains the information of the average temperature, $T_{a v}$ of the system.

In case of vanishing radial flow velocity the inverse slope of the photon and dilepton spectra represent the average temperature, $T_{\mathrm{a} v}$ of the system. However, in case of non-zero radial flow the inverse slope contains the effect of average temperature as well as that of $v_{\mathrm{r}}$. Therefore, the difference in the slopes of the two cases will enable us to estimate the amount of collectivity in the system.

As mentioned before for large initial temperature transverse momentum distribution of photons from QGP dominates over its hadronic counterpart for $p_{T} \geq 1.5 \mathrm{GeV}$. However, in case of dileptons one has to select both the $M$ and the $p_{T}$ windows to observe QGP. For example the thermal dileptons from hadrons dominate over those from QGP for $M \sim 0.75 \mathrm{GeV}$. Therefore, for estimating the radial velocity in the hadronic phase we chose $p_{T} \sim 0.5$ $\mathrm{GeV}$ and $M \sim 0.75 \mathrm{GeV}$ for demonstrative purpose. Similarly a $p_{T}$ and $M$ windows may be selected where contributions from QGP dominates.

The exponential slope of the ratio $\left(C_{3}\right)$ can be related to the individual slopes of photons $\left(T_{\mathrm{e} f f 1}^{-1}\right)$ and dileptons $\left(T_{\mathrm{e} f f 2}^{-1}\right)$ as follows:

$$
C_{3} \times\left(m_{T}-p_{T}\right)=\frac{m_{T}}{T_{e f f 2}}-\frac{p_{T}}{T_{\text {eff } 1}}
$$

writing the effective (blue shifted) temperatures of the photon spectra and dilepton spectra as

$$
\begin{gathered}
T_{e f f 1}=T_{a v} \sqrt{\frac{\left(1+v_{r}\right)}{\left(1-v_{r}\right)}}, \\
T_{e f f 2}=T_{a v}+M v_{r}{ }^{2}
\end{gathered}
$$

we obtain,

$C_{3} \times\left(m_{T}-p_{T}\right)=\frac{m_{T}}{T_{a v}+M v_{r}{ }^{2}}-\frac{p_{T}}{T_{a v} \sqrt{\left(1+v_{r}\right) /\left(1-v_{r}\right)}}$ 


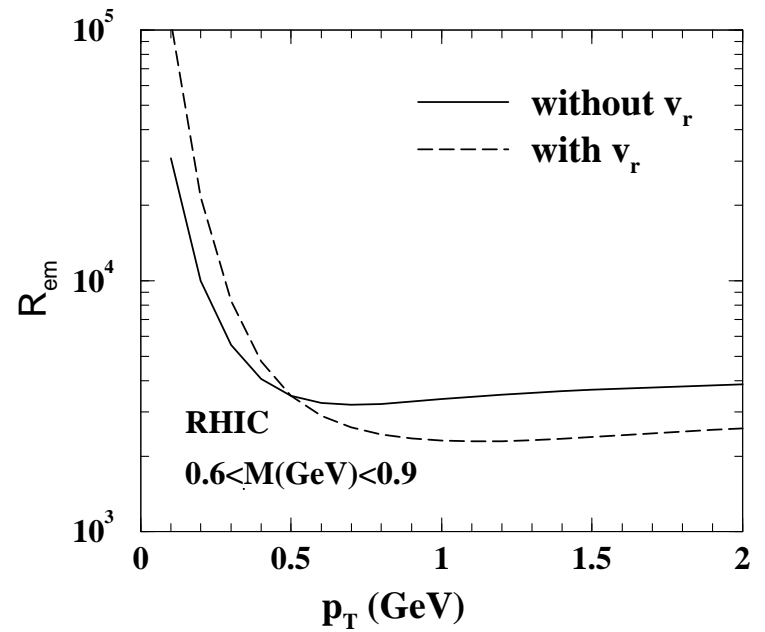

FIG. 2: $R_{\mathrm{e} m}$ as a function of $p_{T}$ with and without radial flow for invariant mass $0.6<M(G e V)<0.9$. The spectra with radial flow is normalized to the one without radial flow at $p_{T}=0.5 \mathrm{GeV}$

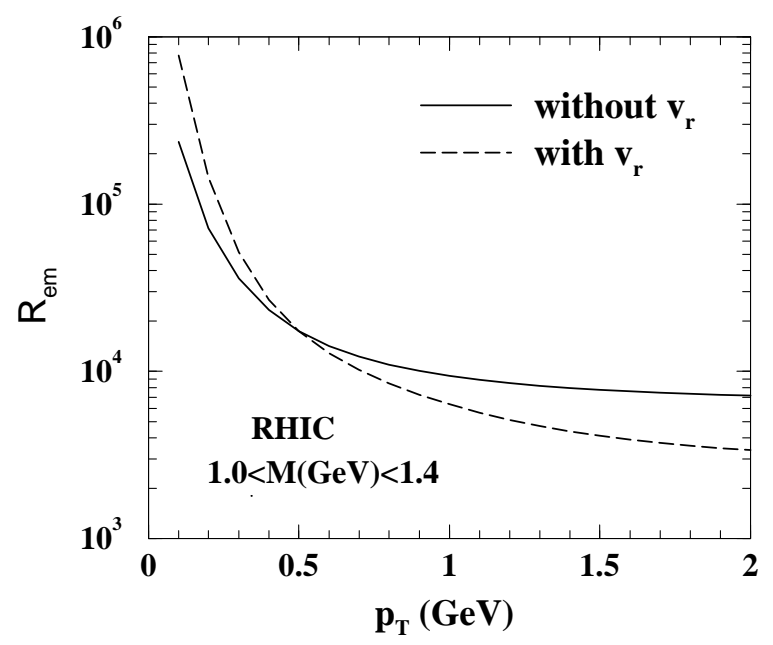

FIG. 3: $R_{\mathrm{e} m}$ as a function of $p_{T}$ as in the above figure for invariant mass $1.0<M(G e V)<1.4$.

Further simplification leads to

$$
a T_{a v}^{2}+b T_{a v}+c=0
$$

where $a, b$ and $c$ are functions of $v_{\mathrm{r}}$. Solving Eq. 66 for a given $C_{3}, M$ and $p_{T}$ we obtain $v_{\mathrm{r}}$ as a function of the average temperature. The results are displayed in Figs. 4 and 5 for initial conditions of RHIC and LHC energies for invariant mass and $p_{T}$ windows indicated. The contributions in the $M$ and $p_{T}$ windows shown in Fig. 4 are dominated by the hadronic phase i.e. from temperature range $T_{c} \sim 192 \mathrm{MeV}$ to $T_{F} \sim 120 \mathrm{MeV}$. The radial velocity increases sharply with decrease in $T_{\mathrm{a} v}$ in the hadronic phase.

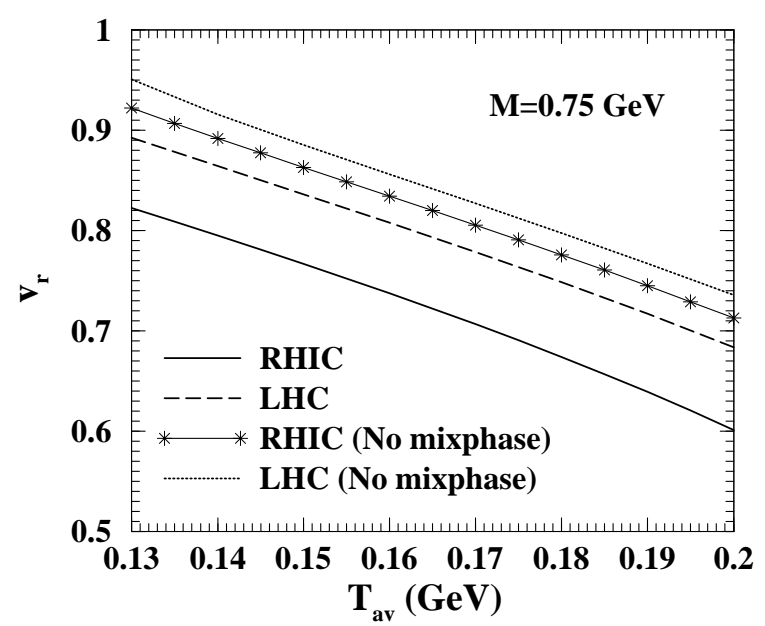

FIG. 4: Variation of $v_{\mathrm{r}}$ with $T_{\mathrm{a} v}$ for $M=0.75 \mathrm{GeV}$ and $p_{T}=0.5 \mathrm{GeV}$. The solid (dashed) line indicate the results for RHIC (LHC) for EoS with first order phase transition. The line with asterisk (dotted line) stands for RHIC (LHC)for an EoS which excludes the mixed phase.

We have evaluated $v_{\mathrm{r}}$ with a (continuous) EoS where the mixed phase does not appear. In this case the $v_{\mathrm{r}}$ is larger than the one obtained for a strong first order phase transition ( Fig. (4). Indicating that the presence of the mixed phase (of hadrons and QGP) characterized by zero sound velocity slowed down the expansion of the system, resulting in a lower radial flow. Therefore, extraction of $v_{\mathrm{r}}$ from experimental data will be useful to understand the nature of the transition.

In Fig. 5 the radial velocity is displayed for (average) temperature range which is dominated by QGP phase. The results indicate a moderate $v_{r}$ for RHIC but a large $v_{r}$ is achieved even in the QGP phase for LHC energies, in fact a fast increase in $v_{\mathrm{r}}$ is observed for $T_{\mathrm{a} v}$ close to the transition temperature in case of LHC. The $v_{\mathrm{r}}$ for LHC is much larger than RHIC because of the longer life time and larger internal pressure of the partonic phase in LHC than RHIC.

In a first order phase transition scenario the QGP formed in heavy ion collisions return back to hadrons through a first order phase transition. The temperature changes continuously from $T_{i}$ to $T_{F}$. We estimate the average values of the radial velocity $\left(v_{\text {isoth }}\right)$ on the constant temperature surfaces determined by the conditions: $T(r, \tau)=T_{S}$, for various values $T_{S}$. The variation of $\left(v_{\text {isoth }}\right)$ with $T_{S}$ is depicted in Fig. 6 both for RHIC and LHC energies. $v_{\text {isoth }}$ for LHC is larger than RHIC because of higher initial temperature and hence internal pressure. In contrast to the results shown in Figs. 4 and [5, the variation of $v_{\text {isoth }}$ 


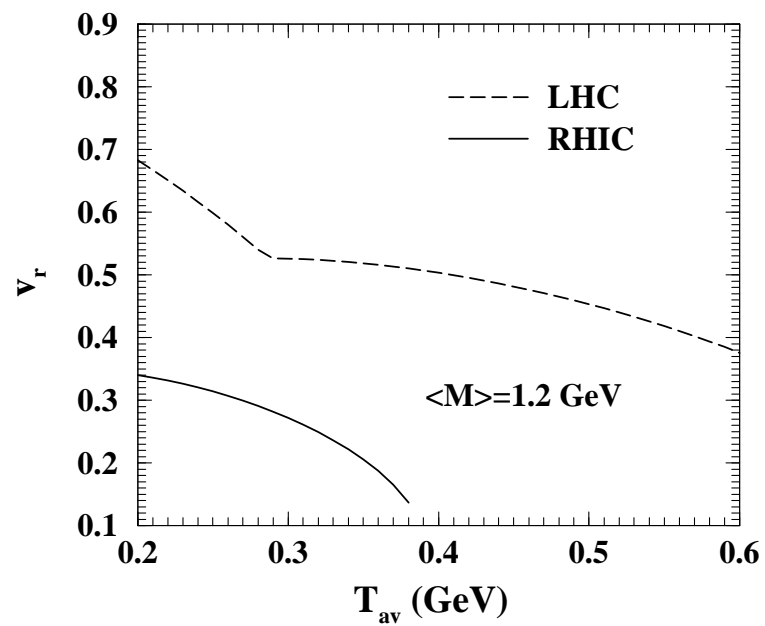

FIG. 5: Variation of $v_{\mathrm{r}}$ with $T_{\mathrm{a} v}$ for $M=1.2 \mathrm{GeV}$ and $p_{T}=0.5 \mathrm{GeV}$ at RHIC and LHC energies for EoS with a first order phase transition.

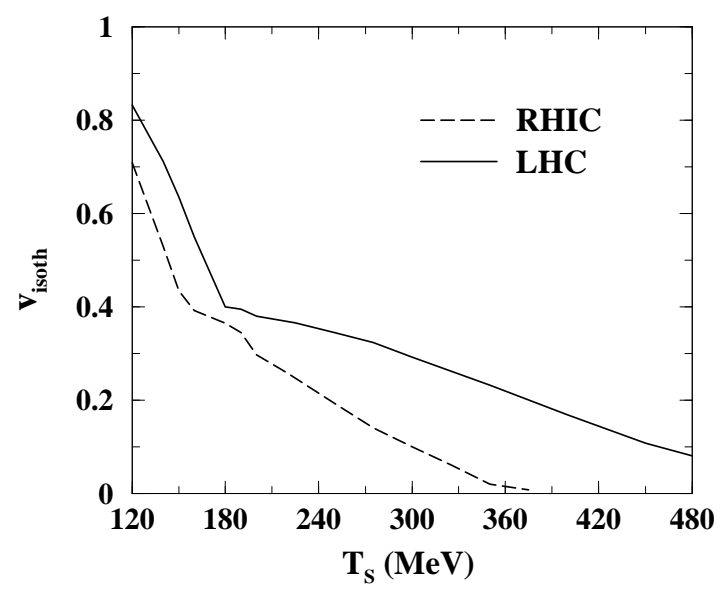

FIG. 6: Variation of average radial velocity of the fluid on the constant temperature surface.

with $T_{S}$ is not measurable as it does not depend on the kinematic variables, $p_{T}$ and $M$. The expansion is slower in the hadronic phase because of the softer EoS as compared to the QGP phase. For given $T_{c}$ and $T_{F}$ the life time of the hadronic phase is larger for softer EoS - allowing the system to develop large radial flow as evident from the results depicted in Fig. 6] for the low temperature part. The effective temperature extracted from the ratio is displayed in Fig. [7] as a function of $M$ for RHIC energy. $T_{\text {eff }}$ increases with $M$ up to the $\rho$-peak and then decreases beyond $\rho$ mass. The reduction of $T_{\mathrm{e} f f}$ beyond $\rho$ is indicating the dominance of the radiation from the high temperature phase in the high $M$ region. For LHC, however, no clear reduction of $T_{\mathrm{e} f f}$ beyond $\rho$
- peak is observed (Fig. 8). At LHC the average temperature and the flow velocity in the early phase (from where large $M$ pairs originate) are large (see Fig. 4). Hence the combination of both large $v_{r}$ and large $T_{a v}$ does not allow $T_{\text {eff }}$ to fall above the $\rho$ peak. The dependence of individual spectra on $T_{F}$ is quite strong, however, we have observed that the slope of the ratio is insensitive to $T_{F}$ and also to $T_{c}$. The slope of the ratio does not change when the parameters like $T_{F}$ changes from $0.120 \mathrm{GeV}$ to 0.150 $\mathrm{GeV}$ and $T_{c}$ from $0.192 \mathrm{GeV}$ to $0.175 \mathrm{GeV}$.

Eliminating $T_{\mathrm{a} v}$ from Eq. 5 and taking the values of $T_{\mathrm{e} f f 1}$ and $T_{\mathrm{e} f f 2}$ from photon and dilepton spectra one can obtain the variation of $v_{r}$ as a function of $M$. The results are shown in Fig. 9 for RHIC and LHC energies. A non-monotonic behaviour of $v_{r}$ with $M$ is observed. A similar nonmonotonic behaviour is observed in the elliptic flow $\left(v_{2}\right)$ of photons as a function of transverse momentum 42, 43]. Comparison of dilepton production from QGP and hadronic sources [17] indicate that in the low $M\left(<m_{\rho}\right)$ and high $M\left(>m_{\phi}\right)$ region the emission rate from QGP dominates over its hadronic counter part if the medium effects on the vector meson spectral functions are neglected. In other words, for a dynamically evolving system the low and high $M$ pairs are emitted from early QGP phase, whereas lepton pairs with $M$ around $\rho$ - mass are emitted from the late hadronic phase. Therefore, low and high $M$ domains represent early time where $v_{\mathrm{r}}$ is low and the $M \sim m_{\rho}$ domain represent late time where $v_{\mathrm{r}}$ is large - giving rise to the observed variation in Fig. 9. indicative of a two different kinds of source in early and late times of the evolving system. For $M \sim 1.2 \mathrm{GeV}$ the flow velocity is not very small since this window is populated by both hadronic and partonic contributions almost equally. Again at LHC energy the partonic phase life time is more which favors the development of larger flow compared to RHIC energy. It is important to note at this point that for LHC, although the slope $C_{3}$ does not show a clear non-monotonic behaviour with $M$, $v_{r}$ does so. Because as described, before the slope $C_{3}$ depends not only on $v_{r}$ but also on $T_{a v}$ and both are large in the partonic phase at LHC.

The two time scales - the life time of the partonic phase $\left(\tau_{\mathrm{Q} G P}\right)$ and the time an inward moving rarefaction wave takes to hit the centre of the cylindrical geometry decide whether radial flow play any important role in the partonic phase or not. The later time scale is defined as $\tau_{\mathrm{r} w} \sim R / c_{s}$ where $R$ is the transverse size of the system and $c_{s}$ is the velocity of sound. If $\tau_{\mathrm{Q} G P} \sim \tau_{\mathrm{r} w}$ then $v_{\mathrm{r}}$ will be large in the partonic phase. Therefore, an increase in $\tau_{i}\left(\tau_{\mathrm{Q} G P} \propto \tau_{i}\right)$ will increase the radial flow in the partonic phase if the initial and the critical temperatures are kept fixed. However, an increase in $\tau_{i}$ from 


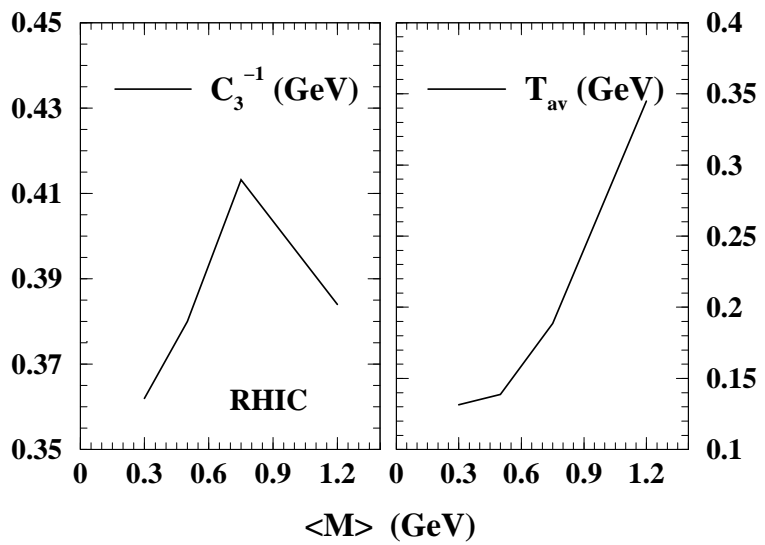

FIG. 7: Left panel: The variation of the slope, $C_{3}$ with invariant mass obtained from the $p_{T}$ spectra of ratio for RHIC energy is displayed in the left panel of the curve. Right panel: the variation of average temperature of the system. The left (right) vertical label is for left (right) panel of the curve

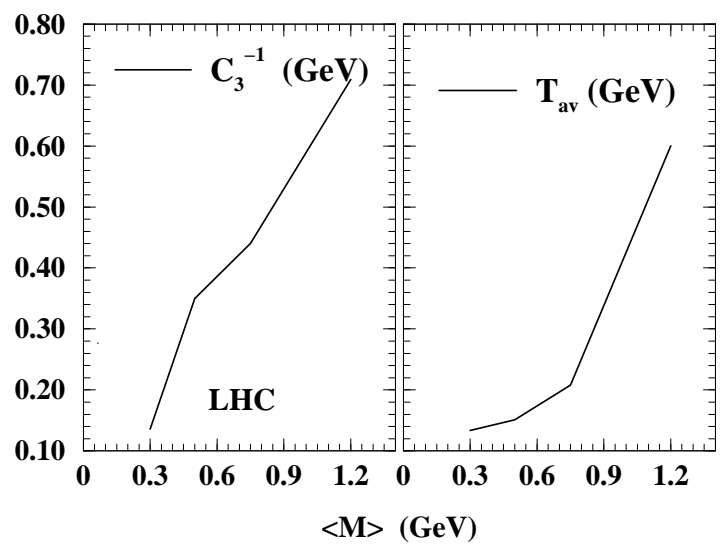

FIG. 8: The variation of the slope, $C_{3}$ with invariant mass obtained from the $p_{T}$ spectra of ratio for LHC. Please note that the scales in the left and the right panels are same.

$\tau_{1}$ to $\tau_{2}$ produces same flow if the $T_{i}$ decreases by a factor $\left(\tau_{2} / \tau_{1}\right)^{1 / 3}$. For a fixed $T_{i}$ an increase in $\tau_{i}$ will increase the effective slope as evident from the right panel of Fig. 10. Therefore, the slope of the ratio may be used effectively to estimate the value of initial thermalization time.

\section{SUMMARY AND DISCUSSIONS}

It has been shown that the $p_{T}$ distribution of thermal photons and lepton pair spectra may be used simultaneously to estimate the magnitude of the ra-

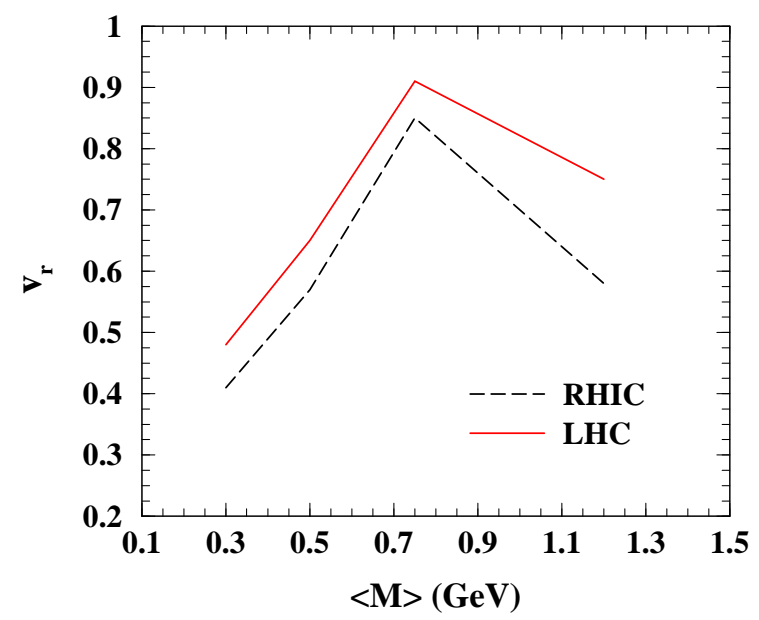

FIG. 9: Radial velocity as a function of $M$ for RHIC and LHC energies.

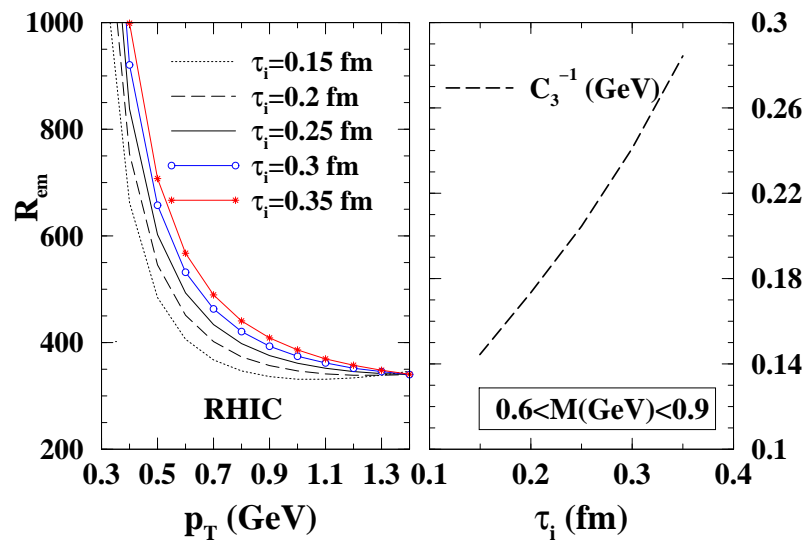

FIG. 10: Left panel: Ratio of the $p_{T}$ spectra for different initial thermalization time $\tau_{i}$ with all other parameters kept same. Right panel: variation of the effective slope $C_{3}$ as a function of initial thermalization time, $\tau_{i}$. The left (right) vertical label is for left (right) panel of the curve

dial velocity of different phases of the matter formed in nuclear collisions at ultra-relativistic energies. Judicious choices of the kinematic variables e.g. the invariant mass and the transverse momentum windows may be selected to estimate the flow velocity in the partonic and hadronic phases of the evolving matter. It has been observed that for RHIC and LHC energies the flow velocity increases with invariant mass up to the $\rho$ peak beyond which it decreases. The $T_{\mathrm{e} f f}$ may not decrease with mass beyond $\rho$ peak if the average temperature and the flow velocity are large in the partonic phase as in case of LHC energy. By doing a simple analysis of photon and dilepton 
spectra we have extracted the radial flow velocity for various invariant mass windows. $v_{\mathrm{r}}$ varies with $M$ non-monotonically. We argue that such a variation indicates the presence of two different types of thermal sources of lepton pairs.
Acknowledgment: JA is supported by DAEBRNS project Sanction No. 2005/21/5-BRNS/2455.
[1] J. C. Collins and M. J. Perry, Phys. Rev. Lett. 34 (1975) 1353.

[2] M. Kisslinger and P. Morley, Phys. Rev. D10 (1976) 2765, 2771.

[3] E. V. Shuryak, Phys. Rep. 61 (1980) 71; Phys. Rep. 115 (1984) 151.

[4] J. Alam, S. Chattopadhyay, T. Nayak, B. Sinha and Y . P. Viyogi (ed), J. Phys. G: Nucl. Part. Phys. 35 (2008) (Proc. Quark Matter 2008).

[5] N. Armesto, N. Armesto, N. Borghini, S. Jeon, U.A. Wiedemann (ed), J. Phys. G: Nucl. Part. Phys. 35 2008.

[6] E. Schnedermann, J. Sollfrank and U. Heinz, Phys. Rev. C 48 (1993) 2462.

[7] C. M. Hung and E. Shuryak, Phys. Rev. C 57 (1998) 1891.

[8] T. Hirano and K. Tsuda, Phys. Rev. C 66 (2002) 054905.

[9] R. Arnaldi et al (NA60 collaboration), Phys. Rev. Lett. 100 (2008) 022302; S. Damjanovic (for NA60 Collaboration), J. Phys. G 35 (2008) 104036.

[10] H van Hees and R. Rapp, Phys. Rev. Lett. 97 102301 (2006).

[11] H van Hees and R. Rapp, Nucl. Phys. A 806339 (2008).

[12] J. Ruppert, C. Gale, T. Renk, P. Lichard and J. I. Kapusta, Phys. Rev. Lett. 100162301 (2008).

[13] T. Renk and J. Ruppert, Phys. ReV. C 77, 024907 (2008).

[14] K. Dusling and I. Zahed, hep-ph0701253

[15] J. Alam, T. Hirano, J. K. Nayak and B. Sinha, arXiv:0902.0446 (nucl-th)

[16] A. Toia (PHENIX collaboration), J. Phys. G 35 (2008) 104307.

[17] J. K. Nayak, J. Alam, S. Sarkar and B. Sinha, Phys. Rev. C 78 (2008) 034903.

[18] L. D. McLerran and T. Toimela, Phys. Rev. D 31, 545 (1985).

[19] C. Gale and J.I. Kapusta, Nucl. Phys. B 357, 65 (1991).

[20] H.A. Weldon, Phys. Rev. D 42, 2384 (1990).

[21] J. Alam, S. Raha and B. Sinha, Phys. Rep. 273, 243 (1996).

[22] J. Kapusta, P. Lichard, and D. Seibert, Phys. Rev. D 44,2774 (1991); R. Bair, H. Nakkagawa, A. Niegawa, and K. Redlich, Z. Phys. C 53, 433(1992);
P. Aurenche, F. Gelis, R. Kobes, and H. Zaraket, Phys. Rev. D 58, 085003 (1998).

[23] E. Braaten and R. D. Pisarski, Nucl. Phys. B 337, 569 (1990); ibid 339, 310 (1990).

[24] P. Arnold, G.D. Moore, and L.G. Yaffe, J. High Energy Phys. 0111, 057 (2001) ; P. Arnold, G.D. Moore, and L.G. Yaffe, J. High Energy Phys. 0112, 009 (2001) ; P. Arnold, G.D. Moore, and L.G. Yaffe, J. High Energy Phys. 0206, 030 (2002).

[25] S. Sarkar, J. Alam, P. Roy , Abhee K. DuttMazumder, B. Dutta-Roy, B. Sinha, Nucl. Phys. A 634 (1998) 208.

[26] P. Roy, S. Sarkar, J. Alam and B. Sinha, Nucl. Phys. A 653 (1999) 277.

[27] S. Turbide, R. Rapp and C. Gale, Phys. ReV. C 69, 014903(2004).

[28] T. Altherr and P. V. Ruuskanen, Nucl. Phys. B 380, 377 (1992).

[29] M. H. Thoma and C. T. Traxler, Phys. ReV. D 56, 198(1997).

[30] J. Alam, S. Sarkar, P. Roy, T. Hatsuda and B. Sinha, Ann. Phys. 286, 159 (2000).

[31] E. V. Shuryak, Rev. Mod. Phys. 651 (1993).

[32] G.E. Brown and M. Rho, Phys. Rep. 269 (1996)333.

[33] R. Rapp and J. Wambach, Adv. Nucl. Phys. 25, $1(2000)$.

[34] H. von Gersdorff, M. Kataja, L. D. McLerran and P. V. Ruskanen, Phys. Rev. D 34 794(1986).

[35] J. D. Bjorken, Phys. Rev. D 27, 140 (1983).

[36] M. Cheng et al., Phys. Rev. D 74, 054507 (2006).

[37] Y. Aoki, Z. Fodor, S. D. Katz and K. K. Szab, Phys. Lett. B 64346 (2006).

[38] B. Mohanty and J. Alam, Phys. Rev. C 68064903 (2003).

[39] B. K. Patra, J. Alam, P. Roy, S. Sarkar and B. Sinha, Nucl. Phys. A 709440 (2002).

[40] J. Alam, J. K Nayak, P. Roy, A. K. Dutt-Mazumder and B. Sinha, J. Phys. G 34871 (2007).

[41] P. Mohanty et al under preparation.

[42] R. Chatterjee, E. S. Frodermann, U. W. Hienz and D. K. Srivastava, Phys. Rev. Lett. 96, 202302 (2006).

[43] F.-M. Liu, T. Hirano, K. Werner and Y. Zhu, arXiv:0906.3566 [hep-ph] 